\title{
On the Galois embedding problem associated to the universal central extension of the alternating group of degree 6
}

\author{
Teresa Crespo * Montserrat Vela ${ }^{\dagger}$ \\ Departament d'Àlgebra i Geometria Departament de Matemàtica Aplicada II \\ Universitat de Barcelona Universitat Politècnica de Catalunya \\ Gran Via de les Corts Catalanes 585 C/Pau Gargallo, 5- Campus Sud- Edifici U \\ E-08007 Barcelona, Spain E-08028 Barcelona, Spain \\ e-mail teresa.crespo@ub.edu e-mail:Montse.Vela@upc.es
}

\begin{abstract}
We obtain an explicit expression for the obstruction to the solvability of the Galois embedding problem associated to the universal central extension of the alternating group of degree 6 .
\end{abstract}

Key words: Galois embedding problem, central simple algebras, Galois descent.

Mathematics Subject Classification 2000: 12F12, 11S20, 11R32, $11 \mathrm{R} 34$.

*Partially supported by grant MTM2009-07024, Spanish Science Ministry.

${ }^{\dagger}$ Partially supported by MECD grant BFM2000-0794-C02-01 and AGAUR 2002SGR 00148 


\section{Introduction}

Let $L \mid K$ be a Galois extension with Galois group the alternating group $A_{6}$. Let $6 A_{6}$ be the nontrivial 6 -fold cover of $A_{6}$. We consider the Galois embedding problem

$$
6 A_{6} \rightarrow A_{6} \simeq \operatorname{Gal}(L \mid K) .
$$

A solution to $(1)$ is a field $\widetilde{L}$, containing $L$, which is a Galois extension of $K$ such that $\operatorname{Gal}(\widetilde{L} \mid K) \simeq$ $6 A_{6}$ and the diagram

$$
\begin{array}{ccc}
\operatorname{Gal}(\widetilde{L} \mid K) & \rightarrow & \operatorname{Gal}(L \mid K) \\
\mid 2 & & \mid 2 \\
6 A_{6} & \rightarrow & A_{6}
\end{array}
$$

commutes, where the epimorphism $\operatorname{Gal}(\widetilde{L} \mid K) \rightarrow \operatorname{Gal}(L \mid K)$ is given by restriction. Our aim is to obtain an explicit expression for the obstruction to the solvability of (1).

It is known that, for $n \neq 6,7$, the nontrivial double cover $2 A_{n}$ is the universal central extension of $A_{n}$, i.e. we have a commutative diagram

$$
\begin{aligned}
& 1 \rightarrow\{ \pm 1\} \rightarrow 2 A_{n} \rightarrow A_{n} \rightarrow 1 \\
& 1 \rightarrow \stackrel{\downarrow}{H} \rightarrow \frac{\downarrow}{A_{n}} \rightarrow A_{n} \rightarrow 1
\end{aligned}
$$

for any central extension $1 \rightarrow H \rightarrow \widetilde{A_{n}} \rightarrow A_{n} \rightarrow 1$.

For $n=6,7$, the universal central extension of $A_{n}$ is

$$
\begin{aligned}
6 A_{n}= & \left\langle g_{1}, \ldots, g_{n-2}, z\right| g_{1}^{3}=g_{i}^{2}=\left(g_{i-1} g_{i}\right)^{3}=\left(g_{j} g_{k}\right)^{2}=z^{3},\left(g_{1} g_{4}\right)^{2}=z, z^{6}=\left[z, g_{t}\right]=1 \\
& 1 \leq i \leq 5,1 \leq j \leq k-1, k \leq 5,(j, k) \neq(1,4), 1 \leq t \leq 5\rangle
\end{aligned}
$$

Given a finite Galois extension $L \mid K$ with Galois group $G$ and an epimorphism of groups $\widetilde{G} \rightarrow G$ with abelian kernel $A$, the obstruction to the solvability for the Galois embedding problem

$$
\widetilde{G} \rightarrow G \simeq \operatorname{Gal}(L \mid K)
$$

is given by $\inf \varepsilon \in H^{2}\left(G_{K}, A\right)$, where $G_{K}$ is the absolute Galois group of $K, \varepsilon \in H^{2}(G, A)$ represents $\widetilde{G}$ and inf: $H^{2}(G, A) \rightarrow H^{2}\left(G_{K}, A\right)$ is the induced morphism between cohomology groups.

In the case of the Galois embedding problem given by the nontrivial double cover of the alternating group $A_{n}, n \geq 4$, over a field $K$ of characteristic different from 2, Serre [4] obtained a formula expressing the obstruction to the solvability in terms of the Hasse-Witt of a trace form and allowing its explicit computation. An explicit formula for the solutions to this embedding problem was obtained by Crespo in [1]. These results were generalized in [3] and [2]. An explicit determination of the obstruction to the solvability of embedding problems with a cyclic kernel of order $>2$ in terms of generalized Clifford algebras was obtained by Vela in [6] together with an explicit construction of their solutions. 


\section{Preliminaries}

The group $6 A_{6}$ is isomorphic to the subgroup of $S L(6, \mathbb{Q}(\omega))$ generated by the two matrices

$$
M_{1}:=-\left(\begin{array}{cccccc}
1 & 0 & 0 & 0 & 0 & 0 \\
0 & 0 & 1 & 0 & 0 & 0 \\
0 & 1 & 0 & 0 & 0 & 0 \\
0 & 0 & 0 & 0 & 1 & 0 \\
0 & 0 & 0 & 1 & 0 & 0 \\
0 & 0 & 0 & 0 & 0 & 1
\end{array}\right), M_{2}:=\left(\begin{array}{cccccc}
0 & 1 & 0 & 0 & 0 & 0 \\
0 & 0 & \omega & 0 & 0 & 0 \\
0 & 0 & 0 & 1 & 0 & 0 \\
\omega^{2} & 0 & 0 & 0 & 0 & 0 \\
0 & 0 & 0 & 0 & 0 & 1 \\
0 & 0 & 0 & 0 & 1 & 0
\end{array}\right),
$$

where $\omega$ denotes a primitive third root of unity, and the epimorphism $6 A_{6} \rightarrow A_{6}$ can be given by

$$
\begin{aligned}
& M_{1} \quad \mapsto \quad \sigma:=(2,3)(4,5) \\
& M_{2} \quad \mapsto \quad \tau:=(1,4,3,2)(5,6)
\end{aligned}
$$

For $K$ a field containing $\mathbb{Q}(\omega)$, we obtain a representation

$$
\rho: A_{6} \rightarrow P G L(6, K)=\operatorname{Aut}\left(M_{6 \times 6}(K)\right)
$$

and a commutative diagram

$$
\begin{array}{cccc}
6 A_{6} & \rightarrow & A_{6} \\
\downarrow & & \downarrow \rho \\
S L(6, K) & \rightarrow & P G L(6, K)
\end{array}
$$

In this situation, we may apply a result of Fröhlich and obtain the following statement.

Proposition 2.1. ([3], App. III) Let us denote by $B$ the twisted algebra of $A:=M_{6 \times 6}(K)$ by the 1-cocycle $\rho: A_{6} \rightarrow A u t\left(M_{6 \times 6}(K)\right)$. The obstruction to the solvability of the embedding problem

$$
6 A_{6} \rightarrow A_{6} \simeq \operatorname{Gal}(L \mid K)
$$

is given by the class of the algebra $B$ in the Brauer group $\operatorname{Br}(K)$ of the field $K$.

\section{Main result}

Let the field $L$ be given as the splitting field of a monic irreducible polynomial of degree 6 in $K[X]$.

Theorem 3.1. The twisted algebra $B$ of $M_{6 \times 6}(K)$ by the 1-cocycle $\rho$ is the subalgebra of $M_{6 \times 6}(L)$ whose elements $E=\left(a_{i j}\right)_{1 \leq i, j \leq 6}$ satisfy

$$
\begin{array}{ll}
a_{i i}=\sum_{k=0}^{5} \lambda_{k} x_{i}^{k}, & \lambda_{k} \in K \\
a_{i j}=z_{i j} \sum_{k=0}^{5} \sum_{l=0}^{4} \mu_{k l} x_{i}^{k} x_{j}^{l}, & \mu_{k l} \in K, i \neq j,
\end{array}
$$

where $x_{i}, 1 \leq i \leq 6$, denote the roots in $L$ of the polynomial $f$ realizing the group $A_{6}$ over $K$ and the elements $z_{i j}$ are given by 


$$
\begin{aligned}
& z_{12}=\left(x_{3}+x_{6}\right)\left(x_{4}+x_{5}\right)+\omega\left(x_{3}+x_{4}\right)\left(x_{5}+x_{6}\right)+\omega^{2}\left(x_{3}+x_{5}\right)\left(x_{4}+x_{6}\right) \\
& z_{13}=\left(x_{2}+x_{6}\right)\left(x_{4}+x_{5}\right)+\omega\left(x_{2}+x_{5}\right)\left(x_{4}+x_{6}\right)+\omega^{2}\left(x_{2}+x_{4}\right)\left(x_{5}+x_{6}\right) \\
& z_{14}=\left(x_{2}+x_{5}\right)\left(x_{3}+x_{6}\right)+\omega\left(x_{2}+x_{6}\right)\left(x_{3}+x_{5}\right)+\omega^{2}\left(x_{2}+x_{3}\right)\left(x_{5}+x_{6}\right) \\
& z_{15}=\left(x_{2}+x_{6}\right)\left(x_{3}+x_{4}\right)+\omega\left(x_{2}+x_{4}\right)\left(x_{3}+x_{6}\right)+\omega^{2}\left(x_{2}+x_{3}\right)\left(x_{4}+x_{6}\right) \\
& z_{16}=\left(x_{2}+x_{3}\right)\left(x_{4}+x_{5}\right)+\omega\left(x_{2}+x_{4}\right)\left(x_{3}+x_{5}\right)+\omega^{2}\left(x_{2}+x_{5}\right)\left(x_{3}+x_{4}\right) \\
& z_{23}=\left(x_{1}+x_{4}\right)\left(x_{5}+x_{6}\right)+\omega\left(x_{1}+x_{6}\right)\left(x_{4}+x_{5}\right)+\omega^{2}\left(x_{1}+x_{5}\right)\left(x_{4}+x_{6}\right) \\
& z_{24}=\left(x_{1}+x_{6}\right)\left(x_{3}+x_{5}\right)+\omega\left(x_{1}+x_{5}\right)\left(x_{3}+x_{6}\right)+\omega^{2}\left(x_{1}+x_{3}\right)\left(x_{5}+x_{6}\right) \\
& z_{25}=\left(x_{1}+x_{6}\right)\left(x_{3}+x_{4}\right)+\omega\left(x_{1}+x_{3}\right)\left(x_{4}+x_{6}\right)+\omega^{2}\left(x_{1}+x_{4}\right)\left(x_{3}+x_{6}\right) \\
& z_{26}=\left(x_{1}+x_{4}\right)\left(x_{3}+x_{5}\right)+\omega\left(x_{1}+x_{3}\right)\left(x_{4}+x_{5}\right)+\omega^{2}\left(x_{1}+x_{5}\right)\left(x_{3}+x_{4}\right) \\
& z_{34}=\left(x_{1}+x_{6}\right)\left(x_{2}+x_{5}\right)+\omega\left(x_{1}+x_{2}\right)\left(x_{5}+x_{6}\right)+\omega^{2}\left(x_{1}+x_{5}\right)\left(x_{2}+x_{6}\right) \\
& z_{35}=\left(x_{1}+x_{6}\right)\left(x_{2}+x_{4}\right)+\omega\left(x_{1}+x_{4}\right)\left(x_{2}+x_{6}\right)+\omega^{2}\left(x_{1}+x_{2}\right)\left(x_{4}+x_{6}\right) \\
& z_{36}=\left(x_{1}+x_{5}\right)\left(x_{2}+x_{4}\right)+\omega\left(x_{1}+x_{2}\right)\left(x_{4}+x_{5}\right)+\omega^{2}\left(x_{1}+x_{4}\right)\left(x_{2}+x_{5}\right) \\
& z_{45}=\left(x_{1}+x_{3}\right)\left(x_{2}+x_{6}\right)+\omega\left(x_{1}+x_{6}\right)\left(x_{2}+x_{3}\right)+\omega^{2}\left(x_{1}+x_{2}\right)\left(x_{3}+x_{6}\right) \\
& z_{46}=\left(x_{1}+x_{3}\right)\left(x_{2}+x_{5}\right)+\omega\left(x_{1}+x_{2}\right)\left(x_{3}+x_{5}\right)+\omega^{2}\left(x_{1}+x_{5}\right)\left(x_{2}+x_{3}\right) \\
& z_{56}=\left(x_{1}+x_{2}\right)\left(x_{3}+x_{4}\right)+\omega\left(x_{1}+x_{3}\right)\left(x_{2}+x_{4}\right)+\omega^{2}\left(x_{1}+x_{4}\right)\left(x_{2}+x_{3}\right)
\end{aligned}
$$

and, for $i<j, z_{j i}$ is obtained by permuting 1 and $\omega^{2}$ in the formula giving $z_{i j}$.

Note that the elements $\lambda_{k}$ and $\mu_{k l}$ do not depend on $i, j$, so we obtain indeed a K-algebra of dimension 36 .

Proof. By Galois descent, the algebra $B$ is the $K$-subalgebra of $M_{6 \times 6}(L) \simeq A \otimes_{K} L$ fixed by the action of the group $G=\operatorname{Gal}(L \mid K)$, where $G$ acts on $A$ via the representation $\varphi$ given by

$$
\begin{array}{rccccc}
\varphi: & A_{6} & \longrightarrow & \operatorname{Aut}(A) & & \\
s & \mapsto & \varphi(s): & A & \rightarrow & A \\
& & & & \mapsto & \widetilde{s} E \widetilde{s}^{-1},
\end{array}
$$

where $\widetilde{s}$ is the preimage of $\rho(s)$ by the epimorphism $S L(6, K) \rightarrow P G L(6, K)$, and $G$ acts on $L$ by the Galois action (see [5] X $\S 2$ ).

If $E=\left(a_{i j}\right)_{1 \leq i, j \leq 6}$ is a matrix in $M_{6 \times 6}(L)$, we obtain

$$
\varphi(\sigma)(E)=M_{1} E M_{1}^{-1}=\left(\begin{array}{cccccc}
a_{11} & a_{13} & a_{12} & a_{15} & a_{14} & a_{16} \\
a_{31} & a_{33} & a_{32} & a_{35} & a_{34} & a_{36} \\
a_{21} & a_{23} & a_{22} & a_{25} & a_{24} & a_{26} \\
a_{51} & a_{53} & a_{52} & a_{55} & a_{54} & a_{56} \\
a_{41} & a_{43} & a_{42} & a_{45} & a_{44} & a_{46} \\
a_{61} & a_{63} & a_{62} & a_{65} & a_{64} & a_{66}
\end{array}\right)
$$

and

$$
\rho(\tau)(E)=M_{2} E M_{2}^{-1}=\left(\begin{array}{cccccc}
a_{22} & \omega^{2} a_{23} & a_{24} & \omega a_{21} & a_{26} & a_{25} \\
\omega a_{32} & a_{33} & \omega a_{34} & \omega^{2} a_{31} & \omega a_{36} & \omega a_{35} \\
a_{42} & \omega^{2} a_{43} & a_{44} & \omega a_{41} & a_{46} & a_{45} \\
\omega^{2} a_{12} & \omega a_{13} & \omega^{2} a_{14} & a_{11} & \omega^{2} a_{16} & \omega^{2} a_{15} \\
a_{62} & \omega^{2} a_{63} & a_{64} & \omega a_{61} & a_{66} & a_{65} \\
a_{52} & \omega^{2} a_{53} & a_{54} & \omega a_{51} & a_{56} & a_{55}
\end{array}\right) .
$$

Hence, the equivalent conditions for a matrix $E=\left(a_{i j}\right)_{1 \leq i, j \leq 6}$ to be fixed under $\sigma$ are 


$$
\begin{array}{llllll}
a_{11}=a_{11}^{\sigma} & a_{12}=a_{13}^{\sigma} & a_{13}=a_{12}^{\sigma} & a_{14}=a_{15}^{\sigma} & a_{15}=a_{14}^{\sigma} & a_{16}=a_{16}^{\sigma} \\
a_{21}=a_{31}^{\sigma} & a_{22}=a_{33}^{\sigma} & a_{23}=a_{32}^{\sigma} & a_{24}=a_{35}^{\sigma} & a_{25}=a_{34}^{\sigma} & a_{26}=a_{36}^{\sigma} \\
a_{31}=a_{21}^{\sigma} & a_{32}=a_{23}^{\sigma} & a_{33}=a_{22}^{\sigma} & a_{34}=a_{25}^{\sigma} & a_{35}=a_{24}^{\sigma} & a_{36}=a_{26}^{\sigma} \\
a_{41}=a_{51}^{\sigma} & a_{42}=a_{53}^{\sigma} & a_{43}=a_{52}^{\sigma} & a_{44}=a_{55}^{\sigma} & a_{45}=a_{54}^{\sigma} & a_{46}=a_{56}^{\sigma} \\
a_{51}=a_{41}^{\sigma} & a_{52}=a_{43}^{\sigma} & a_{53}=a_{42}^{\sigma} & a_{54}=a_{45}^{\sigma} & a_{55}=a_{44}^{\sigma} & a_{56}=a_{46}^{\sigma} \\
a_{61}=a_{61}^{\sigma} & a_{62}=a_{63}^{\sigma} & a_{63}=a_{62}^{\sigma} & a_{64}=a_{65}^{\sigma} & a_{65}=a_{64}^{\sigma} & a_{66}=a_{66}^{\sigma} .
\end{array}
$$

and those to be fixed under $\tau$

$$
\begin{array}{llllll}
a_{11}=a_{22}^{\tau} & a_{12}=\omega^{2} a_{23}^{\tau} & a_{13}=a_{24}^{\tau} & a_{14}=\omega a_{21}^{\tau} & a_{15}=a_{26}^{\tau} & a_{16}=a_{25}^{\tau} \\
a_{21}=\omega a_{32}^{\tau} & a_{22}=a_{33}^{\tau} & a_{23}=\omega a_{34}^{\tau} & a_{24}=\omega^{2} a_{31}^{\tau} & a_{25}=\omega a_{36}^{\tau} & a_{26}=\omega a_{35}^{\tau} \\
a_{31}=a_{42}^{\tau} & a_{32}=\omega^{2} a_{43}^{\tau} & a_{33}=a_{44}^{\tau} & a_{34}=\omega a_{41}^{\tau} & a_{35}=a_{46}^{\tau} & a_{36}=a_{45}^{\tau} \\
a_{41}=\omega^{2} a_{12}^{\tau} & a_{42}=\omega a_{13}^{\tau} & a_{43}=\omega^{2} a_{14}^{\tau} & a_{44}=a_{11}^{\tau} & a_{45}=\omega^{2} a_{16}^{\tau} & a_{46}=\omega^{2} a_{15}^{\tau} \\
a_{51}=a_{62}^{\tau} & a_{52}=\omega^{2} a_{63}^{\tau} & a_{53}=a_{64}^{\tau} & a_{54}=\omega a_{61}^{\tau} & a_{55}=a_{66}^{\tau} & a_{56}=a_{65}^{\tau} \\
a_{61}=a_{52}^{\tau} & a_{62}=\omega^{2} a_{53}^{\tau} & a_{63}=a_{54}^{\tau} & a_{64}=\omega a_{51}^{\tau} & a_{65}=a_{56}^{\tau} & a_{66}=a_{55}^{\tau} .
\end{array}
$$

Let us look at the conditions imposed on the elements in the diagonal.

The element $a_{11}$ is fixed by $\sigma=(2,3)(4,5)$ and $\sigma \tau \sigma \tau^{2}=(2,6,4,3,5)$. So, $a_{11}$ belongs to the field $L^{<\sigma, \sigma \tau \sigma \tau^{2}>}$ which clearly contains $K\left(x_{1}\right)$. Now, the subgroup $\left\langle\sigma, \sigma \tau \sigma \tau^{2}\right\rangle$ of $A_{6}$ has order 60 , so $L^{<\sigma, \sigma \tau \sigma \tau^{2}>}=K\left(x_{1}\right)$. As $a_{11} \in L^{<\sigma, \sigma \tau \sigma \tau^{2}>}=K\left(x_{1}\right)$, we may write $a_{11}=\sum_{i=0}^{5} \lambda_{i} x_{1}^{i}$, for some $\lambda_{i}$ in $K$.

From the relations $a_{44}=a_{11}^{\tau}, a_{33}=a_{44}^{\tau}, a_{22}=a_{33}^{\tau}, a_{55}=a_{44}^{\sigma}, a_{66}=a_{55}^{\tau}$, we obtain

$$
a_{k k}=\sum_{i=0}^{5} \lambda_{i} x_{k}^{i} \quad k=1, \ldots, 6 .
$$

We consider now the elements out of the diagonal.

The element $a_{61}$ is fixed by the subgroup $H$ of $A_{6}$ generated by $\sigma$ and the permutation $(2,4)(3,5)=\sigma \tau^{2} \sigma \tau^{3} \sigma \tau^{3} \sigma \tau^{2} \sigma \tau \sigma$. Let $K_{2}:=L^{H}$; as $H$ has order 4 , the degree of $K_{2}$ over $K$ is 90. The field $K_{2}$ contains clearly the field $K_{1}:=K\left(x_{1}, x_{6}\right)$ which has order 30 over $K$, so $\left[K_{2}: K_{1}\right]=3$. Let us look for a generator of the extension $K_{2} / K_{1}$. We have the following towers of fields

$$
A_{4} \quad \begin{array}{ll} 
& L \\
4 & \\
& \\
3 & K_{2}=L^{H} \\
3 & K_{1}=K\left(x_{1}, x_{6}\right) \\
30 \mid &
\end{array}
$$

The extension $L / K_{1}$ is the splitting field of the polynomial

$$
f_{4}(X)=\frac{f(X)}{\left(X-x_{1}\right)\left(X-x_{6}\right)}=\left(X-x_{2}\right)\left(X-x_{3}\right)\left(X-x_{4}\right)\left(X-x_{5}\right) \in K_{1}[X] .
$$

By the Galois correspondence, the field $K_{2}$ is associated to the subgroup $H_{2}=<\sigma,(2,4)(3,5)>$ and the field $K_{1}$ to the alternating group in the four letters $\{2,3,4,5\}$. Hence, the extension $K_{2} / K_{1}$ is the splitting field of the cubic resolvent of $f_{4}$. We may then write $K_{2}=K_{1}\left(y_{1}, y_{2}, y_{3}\right)$, where 


$$
\begin{aligned}
& y_{1}=\left(x_{2}+x_{3}\right)\left(x_{4}+x_{5}\right) \\
& y_{2}=\left(x_{2}+x_{4}\right)\left(x_{3}+x_{5}\right) \\
& y_{3}=\left(x_{2}+x_{5}\right)\left(x_{3}+x_{4}\right) .
\end{aligned}
$$

have

The Galois group of $\operatorname{Gal}\left(K_{2} / K_{1}\right)$ is generated by $\gamma H_{2}$, where $\gamma=(2,3,4)=\sigma \tau \sigma \tau^{3} \sigma \tau \sigma \tau^{2} \tau$. We

$$
a_{16}^{\gamma}=\omega a_{16}, \quad y_{1}^{\gamma}=y_{3}, \quad y_{2}^{\gamma}=y_{1}, \quad y_{3}^{\gamma}=y_{2} .
$$

Hence the element $z=y_{1}+\omega y_{2}+\omega^{2} y_{3}$ satisfies $z^{\gamma}=\omega z$ and we have $a_{16}=\alpha z$, with $\alpha \in K\left(x_{1}, x_{6}\right)$. We obtain then the expression for $a_{16}$ given in the statement. By using the relations (2) and (3), we obtain the formulas for all elements $a_{i j}, 1 \leq i, j \leq 6, i \neq j$.

\section{References}

[1] T. Crespo, Explicit construction of $\widetilde{A}_{n}$ type fields, J. Algebra 127 (1989), 452-461.

[2] T. Crespo, Embedding Galois Problems and Reduced Norms, Proc. Amer. Math. Soc. 112 (1991), 637-639.

[3] A. Fröhlich, Orthogonal representations of Galois groups, Stiefel-Whitney classes and HasseWitt invariants, J. reine ang. Math. 360 (1985), 84-123.

[4] J-P. Serre, L'invariant de Witt de la forme $\operatorname{Tr}\left(x^{2}\right)$, Comment. Math. Helv. 59 (1984), no. 4, 651676 .

[5] J.P. Serre, Corps locaux (10ème ed.), Hermann (Actualités Scientifiques et Industrielles, 1296), Paris, 1968 .

[6] M. Vela, Explicit solutions of Galois Embedding Problems by Means of Generalized Clifford Algebras, J. Symbolic Computation 30 (2000), 811-842. 